\title{
DESGW: Optical Follow-up of BBH LIGO-Virgo Events with DECam
}

\author{
Robert E. Butler ${ }^{1}$, M. Soares-Santos ${ }^{2}$, J. Annis ${ }^{3}$, K. Herner ${ }^{3}$ \\ ${ }^{1}$ Department of Astronomy, Indiana University, 727 E. Third Street, Bloomington, Indiana \\ 47405, USA \\ ${ }^{2}$ Martin A. Fisher School of Physics, Brandeis University, 415 South Street, Waltham, MA \\ 02453, USA \\ ${ }^{3}$ Fermi National Accelerator Laboratory, P.O. Box 500, Batavia, IL 60510, USA
}

\begin{abstract}
The DESGW program is a collaboration between members of the Dark Energy Survey, the wider astronomical community, and the LIGO-Virgo Collaboration to search for optical counterparts of gravitational wave events, such as those expected from binary neutron star mergers or neutron star-black hole mergers. While binary black hole $(\mathrm{BBH})$ events are not expected to produce an electromagnetic (EM) signature, emission is certainly not impossible. The DESGW program has performed follow-up observations of four BBH events detected by LIGO in order to search for any possible EM counterpart. Failure to find such counterparts is still relevant in that it produces limits on optical emission from such events. This is a review of follow-up results from $\mathrm{O} 1 \mathrm{BBH}$ events and a discussion of the status of ongoing uniform re-analysis of all $\mathrm{BBH}$ events that DESGW has followed up to date.
\end{abstract}

Keywords. Keyword1, keyword2, keyword3, etc.

\section{Introduction}

Are black hole mergers visible? Many observatories are engaged in following up LIGO events in an effort to localize the source and to begin study of the matter involved in the merger. Supermassive black holes often have accretion disks and jets - quasars are among the brightest objects in the universe. Stellar-mass black holes with accretion disks are visible in the x-ray, often remarkably so. Stellar-mass black holes without accretion disks may still be visible as they plow through the galactic interstellar medium, but they will be of magnitude $\sim 22$ in i-band at distance $\sim 100$ pc (Heckler et al. 1996; Chisholm et al. 2003), rendering them invisible at the distances of the LIGO black holes. However there is the possibility of an unexpected optical counterpart, perhaps from the ubiquitous magnetic field induced jet.

Why DES and DECam? The combination of the collecting area of the $4 \mathrm{~m}$ diameter Blanco Telescope at NOAO's Cerro Tololo International Observatory in Chile with the $3 \mathrm{deg}^{2}$ field-of-view of the Dark Energy Camera (DECam; Flaugher et al. 2015) gives us an unrivaled ability to cover large areas of sky to faint magnitudes; we are unique in the Southern hemisphere.

\section{Recap and Updates}

Recap of 01 . During O1, DESGW was able to follow up two events from LIGO (sky maps shown on the left in Figure 1). Among ground-based instruments, DESGW was the most complete in both depth and area covered. Our group and its partners have published on these events (GW150914: Soares-Santos et al. 2016, Annis et al. 2016; GW151226: Cowperthwaite et al. 2016). While the total probability coverage ( $P_{\text {total }}$ in

This document was prepared by DES collaboration using the resources of the Fermi National Accelerator Laboratory (Fermilab), a U.S. Department of Energy, Office of Science, HEP User Facility. Fermilab is managed by Fermi Research Alliance, LLC (FRA), acting under Contract No. DE-AC02-07CH11359. 
Table 1) was quite small for each event in O1, the exercise and analysis were critical for future endeavors in DECam optical follow-up.

Improvements for O2. Between the first and second LIGO observing runs (and continuing throughout O2), the DESGW team made marked improvements in both our difference imaging (diffimg; Kessler et al. 2015) and post-processing pipelines. Both pipelines are described in further detail in Herner et al. (2017).

Difference Imaging. There were two main improvements in the diffimg pipeline over the past year: (1) the ability to run nightly, or "on the fly"; and (2) incorporation of the ability to use templates from after a given event to subtract from that event's search images. We can now image the area at a much later time ( $\gg$ kilonova timescale) and use these as templates. We have now done this using the BLISS Survey (NOAO propID 2017A-0260) for the first three BBH events with good success; we effectively doubled our $\varepsilon_{\text {camera }}$ (see Table 1) for GW150914 with this strategy.

Post-processing. We also completely reworked the post-processing pipeline (postproc). The postproc code now creates a number of plots useful for assessing detections and diffimg efficiency. Two of these plots are shown in Figures 2 and 3. The code also has a new step which checks the outputs of diffimg, allowing the user to quickly and easily diagnose problems in the pipeline.

Update on O2. During O2, we followed up two LIGO triggers (as shown in Figure 1). GW170104 was difficult, since we could only reach a total of $14 \%$ probability. Analysis of that event is ongoing. GW170814 was better for us, since the new smaller probability contours from LIGO+Virgo lay directly in the middle of our survey footprint. As shown in Table 1, we were able to cover $84 \%$ of the final map (it was shifted slightly from the original).
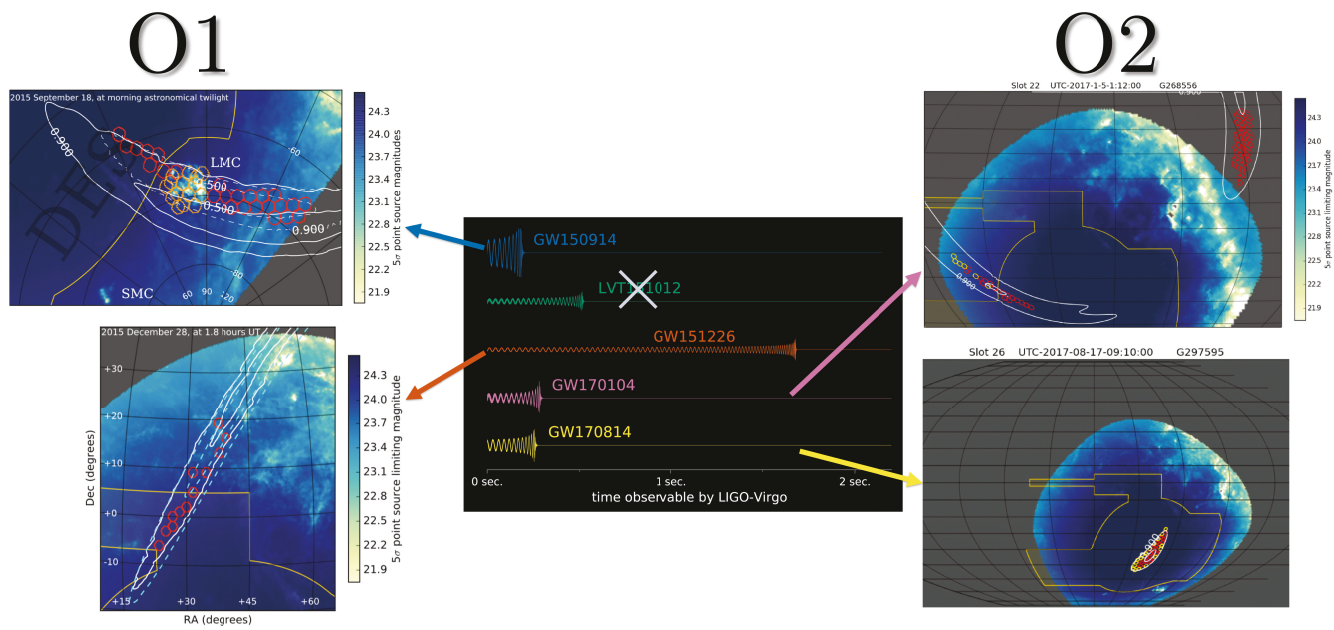

Figure 1. The middle panel (from LIGO's web gallery) shows the fitted chirp signals from each of the five gravitational wave events detected by LIGO so far. The two panels on the left show DESGWs observing plan for the two 2015 events, overlaid with the localization probability maps from LIGO. The two panels on the right show the same for the two 2017 events. Note that GW170814 was also observed by the Virgo detector, and therefore was localized much more precisely. Also note that LIGO did not send a trigger for the marginal LVT151012 event. 


\section{Conclusion}

Although no EM counterparts were detected for any of the four BBH events followed up so far, there is still much to be learned. For our purposes, LIGO events are cumulative. If one wants to place a $95 \%$ confidence limit at a given magnitude, $P_{\text {total }} \geqslant 3$ is necessary. We are well on our way to this goal. With the increased detection rate expected in the next LIGO-Virgo run, it is reasonable to expect this milestone can be reached within that run.

Table 1. For each event, three statistics are given (defined in Annis \& Soares-Santos 2016): (1) $\Sigma_{\text {spatial }}$, the summed probability inside the LIGO spatial localization map covered by the bounding box of images taken; (2) $\varepsilon_{\text {camera }}$, the fraction of the space within the bounding box which is covered with live CCDs, and (3) $\varepsilon_{s k y}$, the fraction of the area imaged that a source would have been visible in (dominated by limiting magnitude). The multiplication of these three gives the probability that the imaging element was able to detect the source: $P_{\text {total }}$.

\begin{tabular}{lcccc}
\hline Event & $\Sigma_{\text {spatial }}$ & $\varepsilon_{\text {camera }}$ & $\varepsilon_{\text {sky }}$ & $P_{\text {total }}$ \\
\hline GW150914 & 0.11 & $0.8 \cdot 0.34$ & 0.8 & .024 \\
GW151226 & 0.02 & 0.8 & 0.8 & .013 \\
GW170104 & 0.14 & 0.96 & $T B D$ & $T B D$ \\
GW170814 & 0.84 & 0.96 & $T B D$ & $T B D$ \\
\hline Total & & & &. .90 $^{*}$
\end{tabular}

*As is shown above, we have not yet made a definite calculation of $\varepsilon_{s k y}$ for the most recent two events, but it is reasonable to expect a value of 0.8 . Using that assumption, we get the total probability given in the bottom of the table.

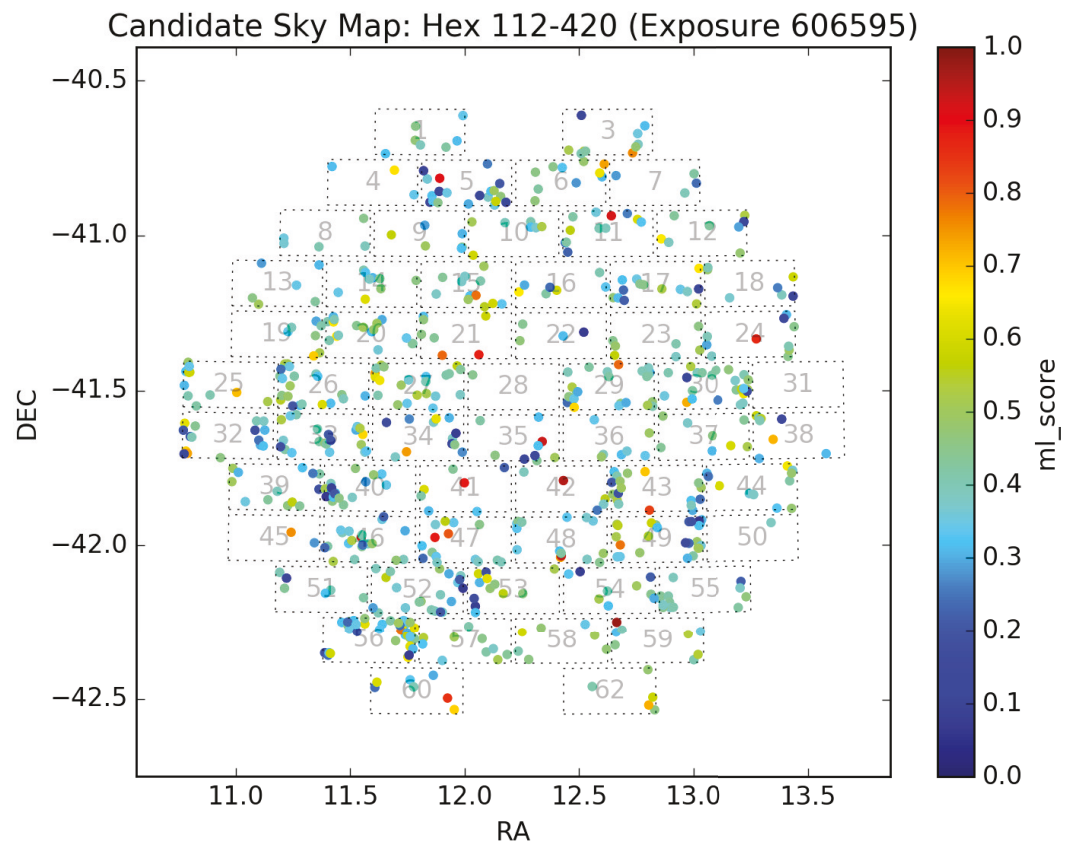

Figure 2. Example hex map output by the postprocessing pipeline. It shows all candidates within a certain exposure, with their associated machine learning scores (these are an assessment of how good the detection was). 


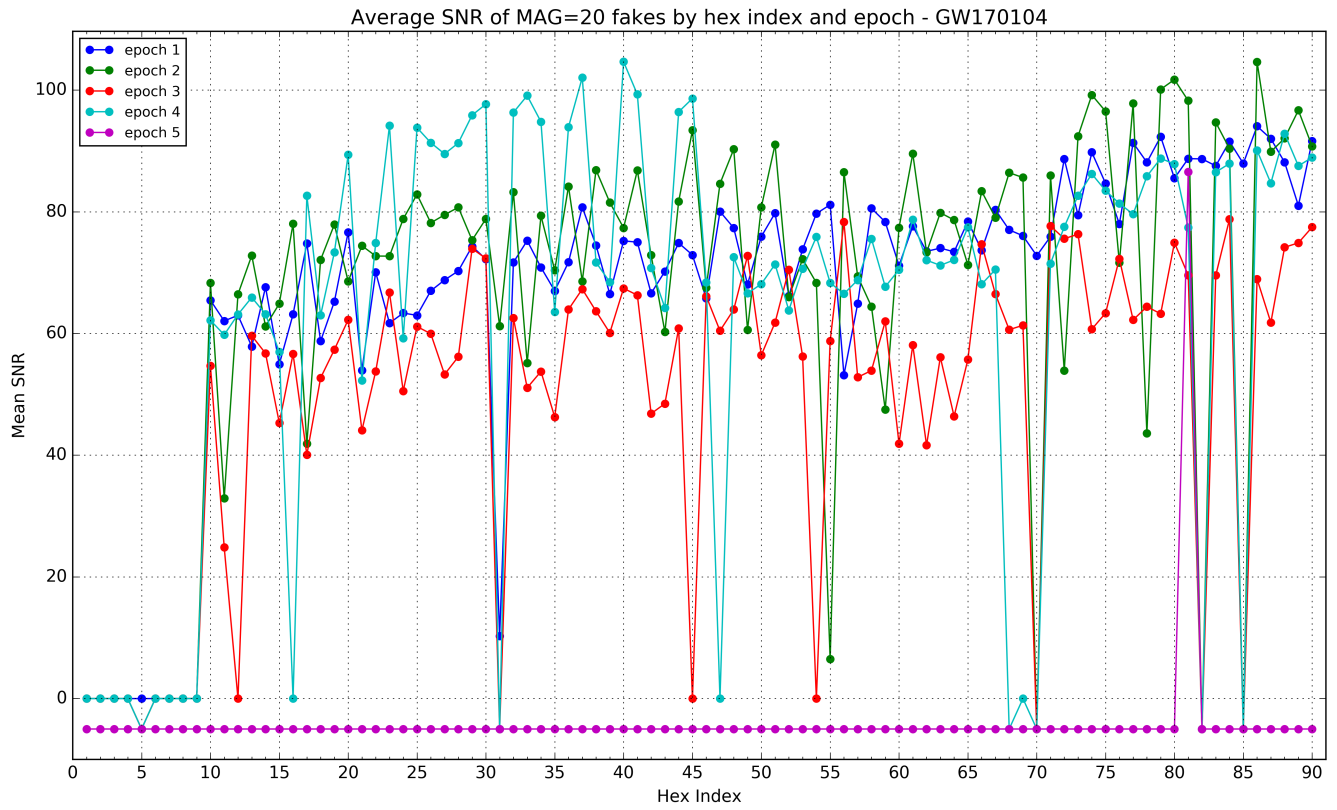

Figure 3. Signal-to-noise ratio (SNR) of artificially implanted signals (fakes) of constant magnitude for the LIGO event GW170104. These artificial transients are arranged on a uniform grid across the sky. The horizontal axis is labeled with indices representing the 90 different pointings on the sky. The vertical axis shows the average SNR of all fakes within a certain pointing, separated into five epochs. For most pointings, four epochs were observed. On this plot, mean SNR values of 0 and -5 are assigned to exposures which have not been processed or taken.).

\section{References}

Annis, J. \& Soares-Santos, M. 2016, PoS (ICHEP2016) 1217

Annis, J., Soares-Santos, M., Berger, E. et al. 2016, ApJ (Letters) 823, L34

Chisholm, J. R., Dodelson, S., \& Kolb, E. W. 2003, ApJ, 596, 437

Cowperthwaite, P. S., Berger, E., Soares-Santos, M., Annis, J. et al. 2016, ApJ (Letters) 826, L29

Flaugher, B., Diehl, H. T., Honscheid, K. et al. 2015, AJ, 150, 150

Heckler, A. F., \& Kolb, E. W. 1996, ApJ (Letters) 472, L85

Herner, K. et al. 2017, J. Phys.: Conf. Ser. 898, 032050

Kessler, R., Marriner, J. et al. 2015, AJ 150, 172

Soares-Santos, M., Kessler, R., Berger, E., Annis, J. et al. 2016, ApJ (Letters) 823, L33 